\title{
Anesthetic Management of an Adolescent With Diamond- Blackfan Syndrome During Posterior Spinal Fusion
}

\author{
Mumin Hakimª, Mahmood Rafiq ${ }^{\mathrm{a}}$, Giorgio Veneziano ${ }^{\mathrm{a}}$, b, Allan C. Beebe ${ }^{\mathrm{c}}$, \\ Jan E. Klamarc, Joseph D. Tobias ${ }^{\mathrm{a}, \mathrm{b}, \mathrm{d}}$
}

\begin{abstract}
Diamond-Blackfan syndrome (DBS) is a rare autosomal dominant ribosomal disorder. Patients present primarily with severe hypoplastic anemia and reticulocytopenia. Due to the craniofacial defects, cardiac pathology, short stature, and skeletal abnormalities, surgical and anesthetic care are frequently required. The associated structural anomalies and end-organ involvement may impact the perioperative care. We present an 18-year-old adolescent with Diamond-Blackfan anemia (DBA) who required anesthetic care for posterior spinal fusion. Previous reports of anesthetic care for these patients are reviewed, the end-organ involvement is discussed, and options for anesthetic care are presented.
\end{abstract}

Keywords: Posterior spinal fusion; Scoliosis; Diamond-Blackfan syndrome; Anemia

\section{Introduction}

Diamond-Blackfan anemia (DBA) was originally described in 1936 by Josephs and was further categorized as a congenital hypoplastic anemia by Diamond and Blackfan in $1938[1,2]$. DBA has also been previously known as congenital hypoplastic anemia, Aase syndrome, and Aase-Smith syndrome II. It is a rare ribosomal disorder with an incidence of $5-7$ per one million live births [3-5]. Familial cases of DBA suggesting autosomal dominant inheritance have been reported while the presence of cytogenetic abnormalities in some patients has led

Manuscript accepted for publication November 11, 2015

aDepartment of Anesthesiology \& Pain Medicine, Nationwide Children's Hospital, Columbus, $\mathrm{OH}$, USA

bDepartment of Anesthesiology, The Ohio State University, Columbus, OH, USA

'Department of Orthopedic Surgery, Nationwide Children's Hospital \& The Ohio State University, Columbus, OH, USA

${ }^{\mathrm{d} C o r r e s p o n d i n g ~ A u t h o r: ~ J o s e p h ~ D . ~ T o b i a s, ~ D e p a r t m e n t ~ o f ~ A n e s t h e s i o l o g y ~ \& ~}$ Pain Medicine, Nationwide Children's Hospital, 700 Children's Drive, Columbus, OH 43205, USA. Email: Joseph.Tobias@nationwidechildrens.org

doi: http://dx.doi.org/10.14740/jmc2370w to the identification of the DBA locus on human chromosome 19. Mutations in the 12 genes that encode ribosomal proteins have been identified in patients with Diamond-Blackfan syndrome (DBS) [6-11].

The initial presentation is generally related to bone marrow dysfunction with severe hypoplastic anemia and reticulocytopenia. Associated involvement in other end-organs may include craniofacial defects, cardiac pathology, short stature, and skeletal abnormalities including scoliosis [12]. Bone marrow involvement is generally limited to the red cell lineage with a normal leukocyte and platelet count. There are associated congenital malformations in approximately $50 \%$ of affected individuals and growth retardation in 30\% [5, 13-15]. Hematologic involvement is noted in $90 \%$ of affected individuals during the first year of life $[16,17]$. Corticosteroid treatment can initially improve the red blood count in approximately $80 \%$ of patients. Eventually, $40 \%$ of affected individuals will be corticosteroid-dependent, $40 \%$ transfusion-dependent while remission occurs in $20 \%[14,18]$. Hematopoietic stem cell transplantation is the only curative therapy for the hematologic manifestations of DBA. Transfusion-related iron overload is the most common complication in transfusion-dependent individuals. DBA is associated with an increased risk for acute myelogenous leukemia, myelodysplastic syndromes, and solid tumors including osteogenic sarcoma. Given the associated congenital anomalies, the need for chronic transfusion therapy, and therapy-related adverse effects, these patients may need anesthetic care during surgery for congenital heart disease, repair of cleft palate, placement of chronic venous devices (Hickman catheter), radiology imaging, and posterior spinal fusion to treat kyphoscoliosis. We present an 18-year-old adolescent with DBA who required anesthetic care for posterior spinal fusion. Previous reports of anesthetic care for these patients are reviewed, the end-organ involvement is discussed, and options for anesthetic care are presented.

\section{Case Report}

Institutional Review Board approval is not required at Nationwide Children's Hospital (Columbus, $\mathrm{OH}$ ) for the presentation of single case reports. The patient was an 18 -year-old $69.9 \mathrm{~kg}$ adolescent who presented for posterior spinal fusion for the treatment of thoracolumbar kyphoscoliosis. His past medi- 


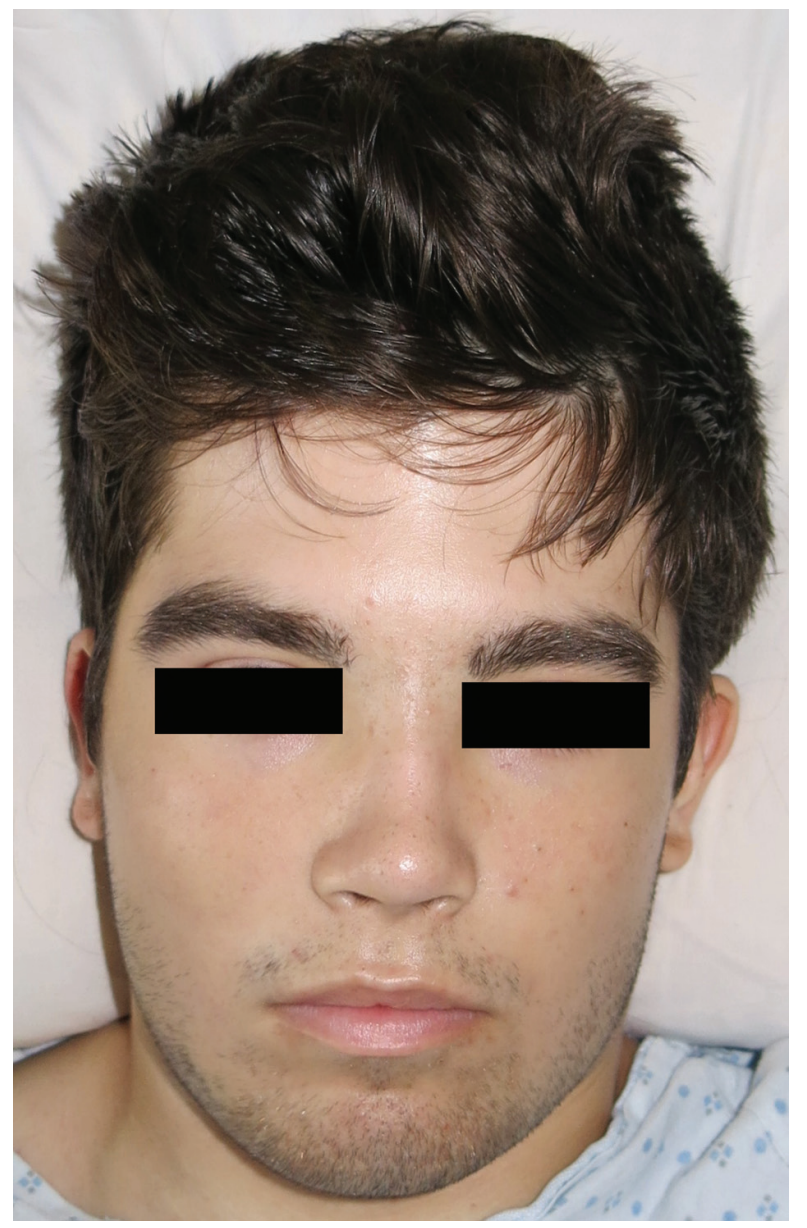

Figure 1. Preoperative photograph of the patient showing macrocephaly, mild hypertelorism, and flattening of nasal bridge which are characteristics of patients with Diamond-Blackfan anemia.

cal history was significant for DBA (steroid responsive) with an RPL11 mutation confirmed by genetic analysis, managed by multiple blood transfusions and corticosteroid therapy. The corticosteroid therapy had been withheld for the past 15 months because of refusal by the family related to concerns of adverse effects. His past surgical history was significant for a repaired partial anomalous pulmonary venous return (PAPVR) at 12 years of age and a history of repeated transfusions of allogeneic packed red blood cells (PRBCs) for the treatment of anemia. Maternal history was significant for macrocytic anemia, thumb anomalies, and heart disease with a murmur. The above-noted gene mutation was present in the mother as well. He was not currently on any chronic home medications. Preoperative physical examination revealed an adolescent in no acute distress with moderate scoliosis, macrocephaly, mild hypertelorism, and flattening of nasal bridge (Fig. 1), a systolic murmur grade II/VI with no signs of congestive cardiac failure, and thumb anomalies. Airway examination was normal including a Mallampati grade I view. Preoperative laboratory evaluation including electrolytes, renal function, coagulation function, blood glucose and hepatic function was normal. The patient has received a transfusion of two units PBRCs 2 days

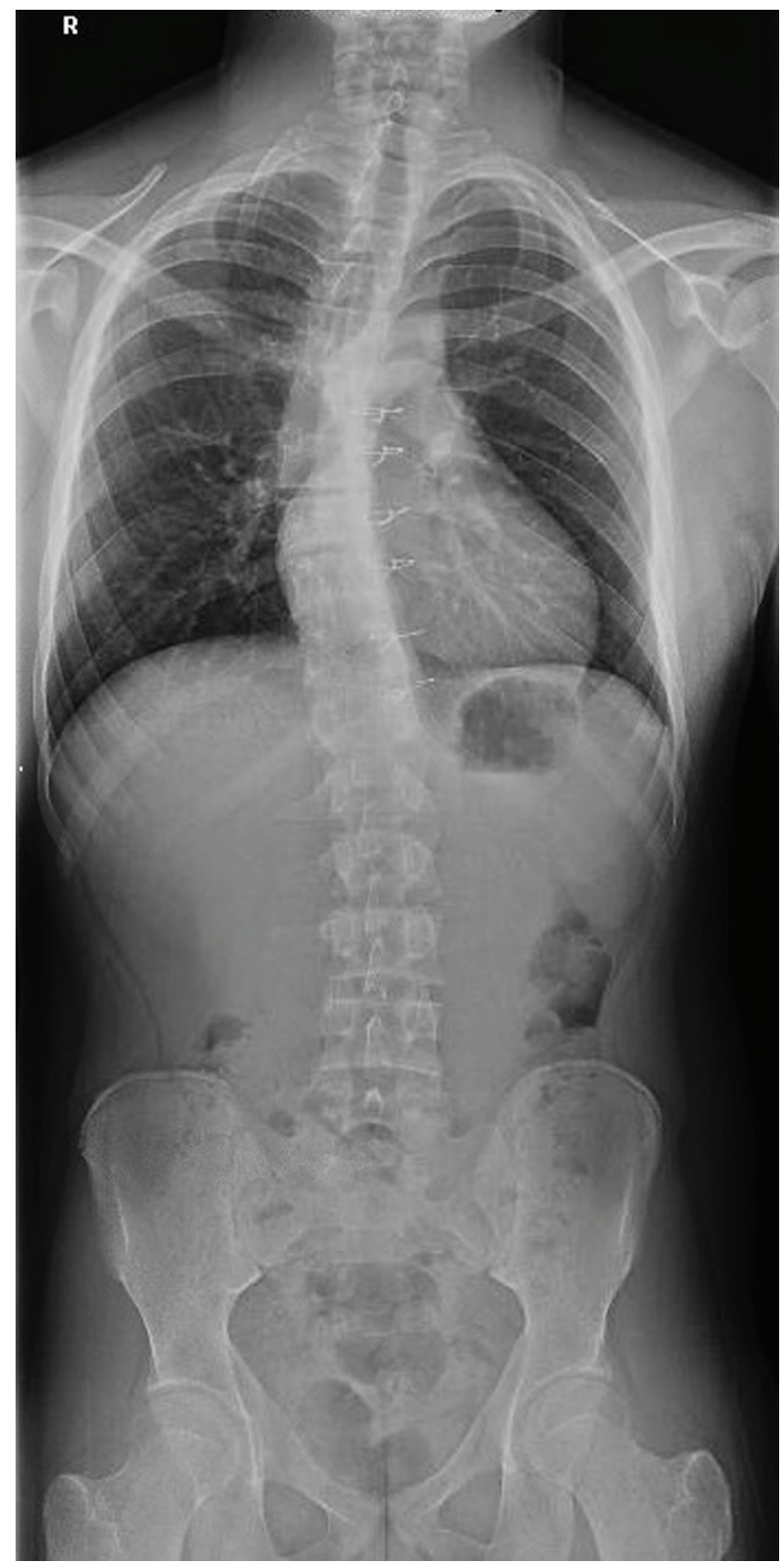

Figure 2. Radiographs of the spine showing severe thoracolumbar kyphoscoliosis.

prior to day of surgery. The post-transfusion hemoglobin was $10.8 \mathrm{~g} / \mathrm{dL}$ with a hematocrit of $32.3 \%$. The MCV was 103.8 and the serum ferritin was $276 \mathrm{ng} / \mathrm{mL}$ (normal range: 31 $294 \mathrm{ng} / \mathrm{mL}$ ). A recent electrocardiogram was normal and the echocardiogram demonstrated normal biventricular function with evidence of the previous repair of the PAPVR without baffle obstruction. Ultrasonography of the kidneys was normal. Radiographs of the spine showed severe thoracolumbar kyphoscoliosis (Fig. 2). Radiographs of both hands showed carpo-metacarpal anomalies consistent with DBS (Fig. 3).

The patient was held nil per os for $8 \mathrm{~h}$ prior to surgery and was transported to the operating room where routine American Society of Anesthesiologists' monitors were applied. An 


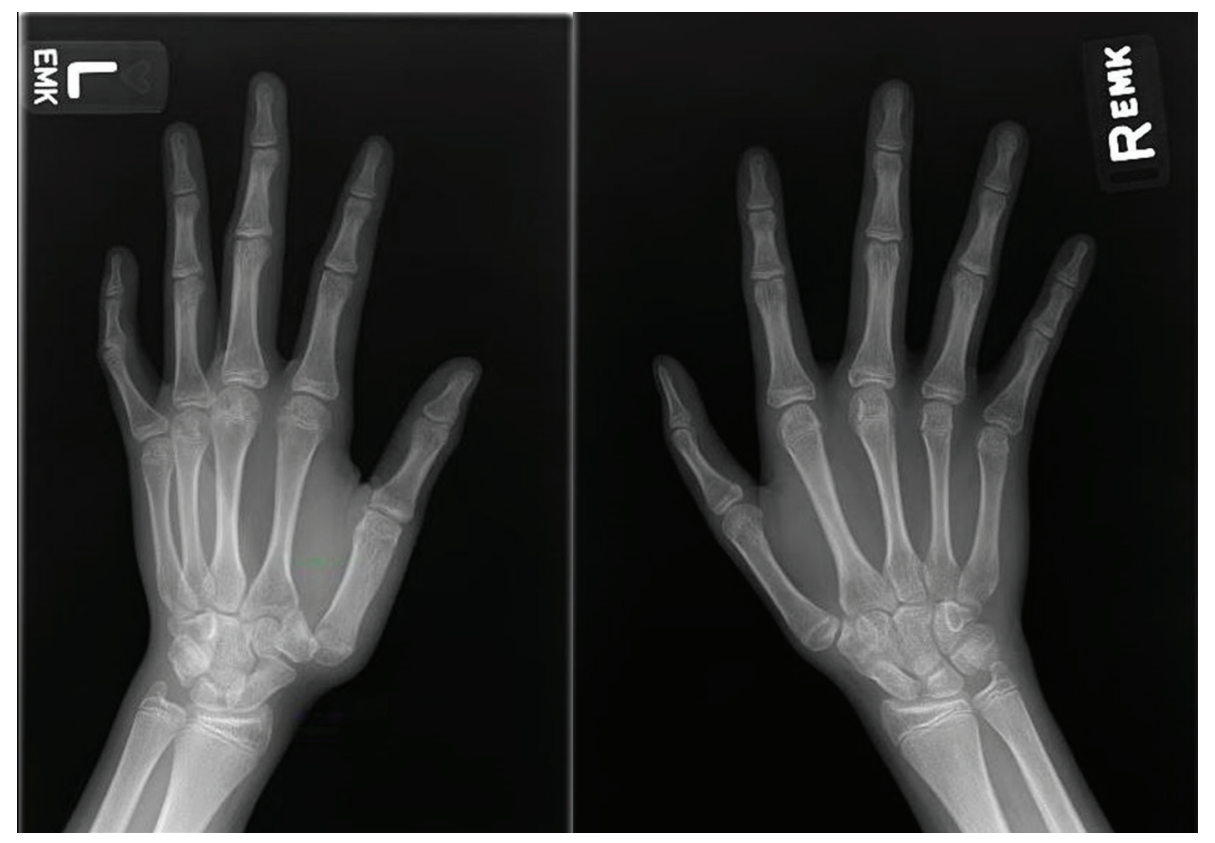

Figure 3. Radiographs of both hands showing carpo-metacarpal anomalies consistent with Diamond-Blackfan anemia.

18-gauge intravenous cannula was placed in the left hand and midazolam $(2 \mathrm{mg})$ administered intravenously prior to anesthetic induction. Anesthesia was induced with propofol (200 $\mathrm{mg}$ ) and lidocaine (100 mg). Bag-valve-mask ventilation was provided without difficulty. Neuromuscular blockade for endotracheal intubation was provided with rocuronium $(50 \mathrm{mg})$ and direct laryngoscopy was performed with a Macintosh 4 blade. Laryngoscopy revealed a Cormack-Lehane grade I view and a $7.0 \mathrm{~mm}$ cuffed endotracheal tube was placed on the first attempt. A 20-gauge arterial cannula was placed in the right radial artery and a second intravenous cannula was placed in the right hand. Tranexamic acid was administered for prevention of fibrinolysis and to limit intraoperative blood loss $(50 \mathrm{mg} /$ $\mathrm{kg}$ bolus dose followed by an infusion at $5 \mathrm{mg} / \mathrm{kg} / \mathrm{h}$ ). Baseline neurophysiological monitoring including motor-evoked potentials (MEP) and somatosensory-evoked potentials (SSEPs) was obtained. The patient was turned and positioned prone. Per our usual practice to allow for neurophysiological monitoring during spinal surgery, anesthesia was maintained with desflurane titrated to maintain the bispectral index (BIS) at 50 - 60 and a sufentanil infusion of $0.3-0.7 \mu \mathrm{g} / \mathrm{kg} / \mathrm{h}$ to maintain the mean arterial pressure at $55-65 \mathrm{~mm} \mathrm{Hg}$. Heart rates varied from 50 to 90 beats per minute with a normal sinus rhythm. Intraoperative fluids included $140 \mathrm{~mL}$ of cell saver autologous blood and $1,285 \mathrm{~mL}$ of isotonic crystalloid solution. No allogeneic blood products were administered. The estimated blood loss was 400 $\mathrm{mL}$. During wound closure, the sufentanil and tranexamic acid infusions were discontinued. Acetaminophen (1,000 mg) was administered intravenously along with incremental doses of hydromorphone to provide postoperative analgesia. Following completion of the surgical procedure, the patient was turned supine and his trachea was extubated when awake. The patient was transferred to the post-anesthesia care unit (PACU) and then admitted to the inpatient surgical ward. Postoperative pain control was provided with hydromorphone delivered via patient-controlled analgesia. The remainder of his postoperative course was uncomplicated and he was discharged home on postoperative day 4 . He did not require any further blood transfusions and his discharge hemoglobin was $9.4 \mathrm{~g} / \mathrm{dL}$.

\section{Discussion}

DBA is a congenital red cell hypoplasia first described by Diamond and Blackfan in $1938[1,2]$. The disease usually presents within the first year of life as an anemia with mild macrocytosis, a low reticulocyte count indicative of bone marrow failure, and the near absence of erythroid precursors in the bone marrow. Recent work has identified the genetic cause to be the disruption of the genes encoding ribosomal proteins [19]. Although RPS19 remains the most commonly mutated gene, responsible for $25 \%$ of DBA cases, our patient had the RPL11 mutation. Only $50 \%$ of DBA patients have known mutations in ribosomal protein genes, leaving the other half of patients with an unknown genetic cause of the disease [11]. The primary clinical feature of DBA is hematologic involvement with a profound isolated normochromic and usually macrocytic anemia. No other cell line is affected as patients have a normal leukocyte and platelet count. Hematologic manifestations occur during the first year of life in $90 \%$ of patients with a median age at presentation of 2 months. Associated congenital malformations are observed in approximately $50 \%$ of affected individuals with more than one abnormality present in up to $25 \%$ of individuals [5, 13-16]. The most commonly reported abnormalities involve: 1) Face and head: microcephaly; ocular hypertelorism; epicanthus, ptosis; broad, flat nasal bridge; microtia, low-set ears; cleft lip/palate, high arched palate; micrognathia; low anterior hairline. 2) Eye: congenital glaucoma, congenital cataract, strabismus. 3) Neck: 
webbed neck, short neck, Klippel-Feil anomaly, Sprengel deformity. 4) Upper limb and hand including thumb: absent radial artery; flat thenar eminence; triphalangeal, duplex, bifid, hypoplastic, or absent thumb. 5) Genitourinary: absent kidney, horseshoe kidney; hypospadias. 6) Heart (15\%): ventricular septal defect, atrial septal defect, coarctation of the aorta, other congenital cardiac anomalies.

Additionally, $30 \%$ of affected individuals will have growth retardation which may be influenced by other factors including chronic corticosteroid treatment. The diagnosis of classic DBA is made when all four of the following diagnostic criteria are met: 1) age younger than 1 year at time of presentation of anemia; 2) macrocytic anemia with no involvement of platelet or leucocytes; 3) low platelet count; 4) normal bone marrow cellularity with decreased erythroid precursors.

Patients with DBA may present for procedures such as repair of cleft palate, radiologic imaging, bone marrow aspiration, placement of central venous access devices, and as noted in our patient for posterior spinal fusion for correction of kyphoscoliosis and surgery for congenital cardiac defects. Effective perioperative care begins with a thorough preoperative evaluation including airway examination, an evaluation of end-organ function which may be affected by DBA, as well as identification of adverse effects related to therapeutic interventions such as long-term corticosteroid treatment. Of primary concern to anesthesia providers is the potential for difficult endotracheal intubation related to craniofacial abnormalities including cleft lip/palate, high arched palate, micrognathia, short neck, and Klippel-Feil anomaly. Although these were not present in our patient and we experienced no difficulties with direct laryngoscopy and endotracheal intubation, such involvement can be seen in up to $50 \%$ of DBA patients. Given these concerns, the ability to accomplish adequate bag-valvemask ventilation should be demonstrated prior to the use of neuromuscular blocking agents. Additionally, the appropriate equipment for dealing with the difficult airway should be readily available including tools for indirect laryngoscopy [20].

As severe anemia is the hallmark of the disease, routine measurement of preoperative hemoglobin is suggested to allow for correction of abnormal parameters prior to surgery and for the preordering of blood products for the day of surgery. Additionally, adequate vascular access should be obtained at the time of surgery to allow for the administration of blood products. Family members should be allowed to donate blood products unless it has been determined that a transplant will not be performed. Leukocyte-filtered blood products should be used to reduce transfusion reactions and limit HLA sensitization in the event that bone marrow transplant is planned. Additionally, as is our routine practice for such orthopedic procedures which may be associated with significant blood loss, various techniques can be employed to limit the need for allogeneic transfusions including maintenance of normothemia, controlled hypotension, the intraoperative use of cell saver, and administration of the anti-fibrinolytic agent, tranexamic acid [21].

Preoperative cardiac evaluation including echocardiography is suggested to evaluate for the presence of associated congenital heart disease as well as to assess myocardial function in the presence of long-term transfusion therapy. Our patient had partial anomalous pulmonary venous return repair with a baffle to left atrium through the superior vena cava at 12 years of age. He currently had no symptoms and echocardiography demonstrated no obstruction to flow through the baffle. Additionally, despite the history of chronic transfusion therapy, he had normal biventricular function.

Iron overload caused by multiple transfusions leads to free iron in heart tissue, which even in low concentrations is toxic to cardiomyocytes. Cardiac iron accumulation usually occurs after organs such as the liver and spleen have become saturated with iron. The most common form of iron injury to the myocardium is congestive cardiomyopathy [22]. Other cardiac pathologies linked to excess iron include pericarditis, coronary artery disease, and conduction defects [23]. Sudden death due to arrhythmias may occur in patients with advanced iron overload [24]. Since cardiac iron burden may increase without clinical symptoms until a critical level is reached, after which systolic function may deteriorate rapidly, preoperative screening with an echocardiogram and electrocardiogram is suggested in patients who are at high risk for iron overload [24, 25].

Chronic RBC transfusion therapy remains necessary in many patients with DBA to prevent the severe complications of bone marrow failure. However, chronic transfusion therapy contributes to iron overload. Successful management and prevention of iron toxicity are dependent on accurate monitoring of patients. Monitoring strategies include invasive tests such as liver biopsy and the direct measurement of iron concentration, which is considered the "gold standard", or imaging studies including magnetic resonance imaging to assess iron loading in the liver and heart or magnetic biosusceptometry (SQUID), which provides a measurement of hepatic iron concentration $[26,27]$. However, these diagnostic tests are invasive, expensive, and subject to sampling errors. As such, routine laboratory monitoring of plasma or serum ferritin concentration in the blood remains the most commonly used test for estimating iron burden [27-31]. This test is inexpensive, non-invasive, and almost universally available. A serum ferritin greater than $200 \mathrm{ng} / \mathrm{mL}$ in premenopausal women and over $300 \mathrm{ng} / \mathrm{mL}$ in men, combined with a fasting transferrin saturation greater than $50 \%$ in women or $60 \%$ in men are sensitive markers of excess iron [29-31]. Serial serum ferritin values greater than $1,000 \mu \mathrm{g} / \mathrm{L}$ are considered the threshold for initiation of iron reduction therapy in transfusion-dependent patients [32].

Chronic iron overload causes progressive damage to the liver, heart, and endocrine glands. In older patients with a history of long-term, chronic transfusion therapy, an evaluation of hepatic function is indicated prior to anesthetic care. Additionally, given the effects of chronic iron overload on pancreatic function, blood glucose monitoring is indicated. Many patients who require frequent transfusions of $\mathrm{RBCs}$ also receive the intravenous chelating agent, deferoxamine, or the oral chelating agent, deferasirox which act by binding free iron in the bloodstream and enhancing its elimination in the urine. Deferasirox affects drug-metabolizing enzymes (CYP3A4), possibly affecting the dose of other concurrent medications [33].

The use of intraoperative neuromonitoring including SSEPs and MEPs further impacts the anesthetic management during PSF $[34,35]$. MEPs are more sensitive to the choice of anesthetic agents than SSEPs [36-38]. Both inhaled and intravenous anesthetic agents depress SSEP and MEP signal attain- 
ment; however, the effect of the inhaled agents was greater at equal minimum alveolar concentrations (MACs) [35]. Therefore, when inhalational anesthetic agents are used, it is generally recommended to maintain end-tidal concentrations levels at or below 0.5 MAC to allow for adequate MEP monitoring. Opioid infusions have little to no effect on neuromonitoring. As has been noted in our previous work, the use of the volatile anesthetic agent, desflurane, in combination with a potent opioid (sufentanil or remifentanil) provides an effective intraoperative anesthetic while allowing for effective neurophysiological monitoring and providing a rapid emergence at the conclusion of the case [39].

Another concern in patients with severe scoliosis related to neuromuscular disease is the potential for postoperative respiratory failure or insufficiency. The progression and severity of scoliosis as measured by the Cobb angle correlate with its impact on respiratory function. As the degree of curvature progresses, scoliosis impedes the movement of the ribs, and places the respiratory muscles at a mechanical disadvantage with a decrease in the compliance of the chest wall directly and the lung indirectly [40]. As the Cobb angle (lateral curvature) progresses to greater than $65^{\circ}$, lung volumes are reduced and ventilation/perfusion mismatch can be observed. In severe cases $\left(\mathrm{Cobb}\right.$ angle $>100^{\circ}$ ), pulmonary hypertension and right ventricular hypertrophy may develop [40]. Scoliosis surgery produces an immediate and transient decrease in vital capacity of up to $40 \%$ in virtually all patients even those with idiopathic scoliosis without co-morbid conditions [41]. These factors may result in postoperative respiratory insufficiency or failure with the need for prolonged postoperative mechanical ventilation. Patients who have abnormal results on their pulmonary function test, particularly a forced vital capacity of less than $30 \%$, or those who have hypercapnia preoperatively have been shown to have a higher need for postoperative ventilation [42]. Given these concerns, preoperative optimization of pulmonary function is suggested including the aggressive treatment of respiratory infections and instructions regarding the use of techniques such as incentive spirometry. Non-invasive techniques of respiratory support such as BiPAP may be used to facilitate postoperative tracheal extubation and avoid postoperative respiratory insufficiency or failure in patients with preexisting pulmonary dysfunction [43, 44].

As corticosteroids are the main stay of therapy in DBA, possible adverse effects of such therapy may include hyperglycemia, peptic ulcer disease, hypokalemia, myopathy, and infections. The incidence of steroid-induced adrenal crisis in children is very low, thus routine hydrocortisone supplementation is generally not necessary prior to short procedures, but may be required perioperatively in patients undergoing prolonged surgical procedures who have been chronically receiving supra-physiologic doses of corticosteroids [45].

In summary, we present a case of DBA, a rare ribosomal genetic disorder characterized by bone marrow failure with severe macrocytic anemia, congenital cardiac defects, and skeletal abnormalities. The bone marrow failure is generally treated by the administration of corticosteroids or multiple transfusions when the patient is not responsive to corticosteroids. Specific perioperative comorbid concerns include the associated underlying anemia, the potential for associated congenital cardiac disease, long-term corticosteroid use, and the presence of facial abnormalities or cleft palate which may lead to problematic airway management.

\section{References}

1. Nathan DG, Clarke BJ, Hillman DG, Alter BP, Housman DE. Erythroid precursors in congenital hypoplastic (Diamond-Blackfan) anemia. J Clin Invest. 1978;61(2):489498.

2. Diamond LK, Blackfan KD. Hypoplastic anemia. Am J Dis Child. 1938;56:464-467.

3. Ball SE, McGuckin CP, Jenkins G, Gordon-Smith EC. Diamond-Blackfan anaemia in the U.K.: analysis of 80 cases from a 20-year birth cohort. Br J Haematol. 1996;94(4):645-653.

4. Campagnoli MF, Garelli E, Quarello P, Carando A, Varotto S, Nobili B, Longoni D, et al. Molecular basis of Diamond-Blackfan anemia: new findings from the Italian registry and a review of the literature. Haematologica. 2004;89(4):480-489.

5. Willig TN, Niemeyer CM, Leblanc T, Tiemann C, Robert A, Budde J, Lambiliotte A, et al. Identification of new prognosis factors from the clinical and epidemiologic analysis of a registry of 229 Diamond-Blackfan anemia patients. DBA group of Societe d'Hematologie et d'Immunologie Pediatrique (SHIP), Gesellshaft fur Padiatrische Onkologie und Hamatologie (GPOH), and the European Society for Pediatric Hematology and Immunology (ESPHI). Pediatr Res. 1999;46(5):553-561.

6. Cmejla R, Cmejlova J, Handrkova H, Petrak J, Pospisilova D. Ribosomal protein S17 gene (RPS17) is mutated in Diamond-Blackfan anemia. Hum Mutat. 2007;28(12):11781182.

7. Doherty L, Sheen MR, Vlachos A, Choesmel V, O'Donohue MF, Clinton C, Schneider HE, et al. Ribosomal protein genes RPS10 and RPS26 are commonly mutated in Diamond-Blackfan anemia. Am J Hum Genet. 2010;86(2):222-228.

8. Farrar JE, Nater M, Caywood E, McDevitt MA, Kowalski J, Takemoto CM, Talbot CC, Jr., et al. Abnormalities of the large ribosomal subunit protein, Rpl35a, in DiamondBlackfan anemia. Blood. 2008;112(5):1582-1592.

9. Gazda HT, Grabowska A, Merida-Long LB, Latawiec E, Schneider HE, Lipton JM, Vlachos A, et al. Ribosomal protein S24 gene is mutated in Diamond-Blackfan anemia. Am J Hum Genet. 2006;79(6):1110-1118.

10. Gazda HT, Sheen MR, Vlachos A, Choesmel V, O'Donohue MF, Schneider H, Darras N, et al. Ribosomal protein L5 and L11 mutations are associated with cleft palate and abnormal thumbs in Diamond-Blackfan anemia patients. Am J Hum Genet. 2008;83(6):769-780.

11. Boria I, Garelli E, Gazda HT, Aspesi A, Quarello P, Pavesi E, Ferrante D, et al. The ribosomal basis of DiamondBlackfan Anemia: mutation and database update. Hum Mutat. 2010;31(12):1269-1279.

12. Dianzani I, Garelli E, Ramenghi U. Diamond-Blackfan Anaemia: an overview. Paediatr Drugs. 2000;2(5):345- 
355.

13. Lipton JM, Atsidaftos E, Zyskind I, Vlachos A. Improving clinical care and elucidating the pathophysiology of Diamond Blackfan anemia: an update from the Diamond Blackfan Anemia Registry. Pediatr Blood Cancer. 2006;46(5):558-564.

14. Vlachos A, Ball S, Dahl N, Alter BP, Sheth S, Ramenghi $\mathrm{U}$, Meerpohl J, et al. Diagnosing and treating Diamond Blackfan anaemia: results of an international clinical consensus conference. Br J Haematol. 2008;142(6):859-876.

15. Vlachos A, Muir E. How I treat Diamond-Blackfan anemia. Blood. 2010;116(19):3715-3723.

16. Bagby GC, Lipton JM, Sloand EM, Schiffer CA. Marrow failure. Hematology Am Soc Hematol Educ Program. 2004:318-336.

17. Ohga S, Mugishima H, Ohara A, Kojima S, Fujisawa K, Yagi K, Higashigawa M, et al. Diamond-Blackfan anemia in Japan: clinical outcomes of prednisolone therapy and hematopoietic stem cell transplantation. Int J Hematol. 2004;79(1):22-30.

18. Chen S, Warszawski J, Bader-Meunier B, Tchernia G, Da Costa L, Marie I, Dommergues JP. Diamond-blackfan anemia and growth status: the French registry. J Pediatr. 2005;147(5):669-673.

19. Draptchinskaia N, Gustavsson P, Andersson B, Pettersson M, Willig TN, Dianzani I, Ball S, et al. The gene encoding ribosomal protein S19 is mutated in Diamond-Blackfan anaemia. Nat Genet. 1999;21(2):169-175.

20. Engelhardt T, Weiss M. A child with a difficult airway: what do I do next? Curr Opin Anaesthesiol. 2012;25(3):326332.

21. Tobias JD. Strategies for minimizing blood loss in orthopedic surgery. Semin Hematol. 2004;41(1 Suppl 1):145156.

22. Liu P, Olivieri N. Iron overload cardiomyopathies: new insights into an old disease. Cardiovasc Drugs Ther. 1994;8(1):101-110.

23. Buja LM, Roberts WC. Iron in the heart. Etiology and clinical significance. Am J Med. 1971;51(2):209-221.

24. Jaeger M, Aul C, Sohngen D, Germing U, Schneider W. [Secondary hemochromatosis in polytransfused patients with myelodysplastic syndromes]. Beitr Infusionsther. 1992;30(464-468.

25. Culshaw V, Yule M, Lawson R. Considerations for anaesthesia in children with haematological malignancy undergoing short procedures. Paediatr Anaesth. 2003;13(5):375383.

26. Cappellini MD, Piga A. Current status in iron chelation in hemoglobinopathies. Curr Mol Med. 2008;8(7):663-674.

27. Perifanis V, Economou M, Christoforides A, Koussi A, Tsitourides I, Athanassiou-Metaxa M. Evaluation of iron overload in beta-thalassemia patients using magnetic resonance imaging. Hemoglobin. 2004;28(1):45-49.

28. Shander A, Sazama K. Clinical consequences of iron overload from chronic red blood cell transfusions, its diagnosis, and its management by chelation therapy. Transfusion. 2010;50(5):1144-1155.

29. Brittenham GM, Cohen AR, McLaren CE, Martin MB, Griffith PM, Nienhuis AW, Young NS, et al. Hepatic iron stores and plasma ferritin concentration in patients with sickle cell anemia and thalassemia major. Am J Hematol. 1993;42(1):81-85.

30. Vichinsky E. Consensus document for transfusion-related iron overload. Semin Hematol. 2001;38(1 Suppl 1):2-4.

31. Borwein S, Ghent CN, Valberg LS. Diagnostic efficacy of screening tests for hereditary hemochromatosis. Can Med Assoc J. 1984;131(8):895-901.

32. Porter JB. Practical management of iron overload. Br J Haematol. 2001;115(2):239-252.

33. Skerjanec A, Wang J, Maren K, Rojkjaer L. Investigation of the pharmacokinetic interactions of deferasirox, a once-daily oral iron chelator, with midazolam, rifampin, and repaglinide in healthy volunteers. J Clin Pharmacol. 2010;50(2):205-213.

34. Deiner S. Highlights of anesthetic considerations for intraoperative neuromonitoring. Semin Cardiothorac Vasc Anesth. 2010;14(1):51-53.

35. Sloan TB, Heyer EJ. Anesthesia for intraoperative neurophysiologic monitoring of the spinal cord. J Clin Neurophysiol. 2002;19(5):430-443.

36. Balvin MJ, Song KM, Slimp JC. Effects of anesthetic regimens and other confounding factors affecting the interpretation of motor evoked potentials during pediatric spine surgery. Am J Electroneurodiagnostic Technol. 2010;50(3):219-244.

37. Reinacher PC, Priebe HJ, Blumrich W, Zentner J, Scheufler KM. The effects of stimulation pattern and sevoflurane concentration on intraoperative motor-evoked potentials. Anesth Analg. 2006;102(3):888-895.

38. Lo YL, Dan YF, Tan YE, Nurjannah S, Tan SB, Tan CT, Raman S. Intra-operative monitoring in scoliosis surgery with multi-pulse cortical stimuli and desflurane anesthesia. Spinal Cord. 2004;42(6):342-345.

39. Martin DP, Bhalla T, Thung A, Rice J, Beebe A, Samora W, Klamar J, et al. A preliminary study of volatile agents or total intravenous anesthesia for neurophysiological monitoring during posterior spinal fusion in adolescents with idiopathic scoliosis. Spine (Phila $\mathrm{Pa}$ 1976). 2014;39(22):E1318-1324.

40. Koumbourlis AC. Scoliosis and the respiratory system. Paediatr Respir Rev. 2006;7(2):152-160.

41. Vedantam R, Lenke LG, Bridwell KH, Haas J, Linville DA. A prospective evaluation of pulmonary function in patients with adolescent idiopathic scoliosis relative to the surgical approach used for spinal arthrodesis. Spine (Phila Pa 1976). 2000;25(1):82-90.

42. Almenrader N, Patel D. Spinal fusion surgery in children with non-idiopathic scoliosis: is there a need for routine postoperative ventilation? Br J Anaesth. 2006;97(6):851857.

43. Jaber S, Michelet P, Chanques G. Role of non-invasive ventilation (NIV) in the perioperative period. Best Pract Res Clin Anaesthesiol. 2010;24(2):253-265.

44. Pelosi P, Jaber S. Non-invasive respiratory support in the perioperative period. Curr Opin Anaesthesiol. 2010;3:2233-28.

45. Kohl BA, Schwartz S. How to manage perioperative endocrine insufficiency. Anesthesiol Clin. 2010;28(1):139-155. 